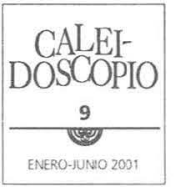

\title{
La explosión demográfica. La comprensión de los procesos de crecimiento geométrico
}

FELIPE MARTINEZ RIZO

Departamento de Educación/UAA

INTRODUCCIÓN

$\mathbf{P}$ ara la inmensa mayoría de las personas, y más para los jóvenes, resulta difícil comprender las cifras actuales de población de los diversos países y ciudades del mundo, dimensionándolas y ubicándolas comparativamente. Especial dificultad representa entender la evolución a lo largo del tiempo de la población, pese a tratarse de un fenómeno que pueden explicarse con unas cuantas variables, y mediante modelos matemáticos relativamente simples, dada la deficiente preparación que suele ofrecer la escuela en lo relativo a los conceptos básicos de la estadística y también a las nociones de crecimiento aritmético y geométrico. La importancia de la demografía, como una de las variables fundamentales que subyacen a cualquier problema social, como los de salud y educación, hace conveniente que estos fenómenos se entiendan mejor. Por ello el propósito de este trabajo es presentar datos básicos sobre la población en las diversas regiones y países del mundo a lo largo de la historia, así como ideas elementales para entender los fenómenos de crecimiento geométrico, en contraposición a los de crecimiento aritmético simple. 


\section{LA EVOLUCIÓN DE LA POBLACIÓN MUNDIAL EN LA HISTORIA}

Las cifras que manejan diversos autores sobre la población humana en diversas regiones del planeta en épocas antiguas son diversas y dudosas, lo que resulta explicable, puesto que no se dispone de registros censales similares a los actuales, sino sólo de información muy incompleta. La imprecisión es mayor, por supuesto, en lo que se refiere a épocas prehistóricas, en cuyo caso los datos se basan en inferencias más o menos aventuradas a partir del estudio de algunos sitios paleoantropológicos y de estimaciones de la densidad posible en ciertos ecosistemas, extrapolando datos de poblaciones actuales que tienen sistemas de vida similares a los que prevalecían en el pasado, basados en la recolección, la cacería o la pesca.

Las estimaciones sobre la población mundial en los inicios de la agricultura, unos 10,000 años antes de la era cristiana, van de uno a cinco millones de habitantes.' A lo largo de varios milenios, y mediante un proceso lento e incierto, la población llegó a unos 150 millones a principios del siglo v antes de Cristo, antes de los momentos cumbre de la civilización griega. A partir de entonces, las estimaciones se vuelven progresivamente menos imprecisas, primero para China, conocida por la antigüedad de censos confiables, y luego para los países europeos.

Se calcula que la población mundial llegó a unos 250 millones de habitantes a principios de la era cristiana, con el apogeo del Imperio Romano y la consolidación del crecimiento chino, que hicieron que cada uno de esos grandes conjuntos contara poco más de 50 millones de habitantes. ${ }^{2}$ Con un máximo poco superior unos

1 Cfr. Sauvy, en Pierre Chaunu, Historia y población. Un futuro sin porvenir. México, FCE, 1982, pág. 51; y Jacques Vallín, La población mundial, Madrid, Alianza, 1995, pág. 63.

2 Chaunu, op. cil., pág. 52. 
dos siglos después, las condiciones ambientales y sociales menos favorables de los siglos siguientes trajeron consigo una reducción que llevó a un mínimo, para el primer milenio de nuestra era, de unos 200 millones de habitantes hacia el inicio del siglo VII. ${ }^{3}$

A partir de entonces inicia un período de crecimiento que lleva la población a un nuevo máximo de unos 442 millones de personas hacia el año 1340. Probablemente mejores condiciones climáticas influyeron en lo anterior; en todo caso, las hambrunas fueron disminuyendo. ${ }^{4} \mathrm{El}$ crecimiento se vio bruscamente interrumpido en Europa, a partir de 1347, con la irrupción de la peste negra; la cristiandad, que contaba unos 65 millones de habitantes en 1340, descendió a algo menos de 40 en 1420. Asia había sido afectada antes por la peste y hambrunas catastróficas, que hicieron que la población china pasara de unos 123 millones hacia el año 1200, a sólo unos 60 en $1381 .{ }^{5}$ En conjunto, la población total del planeta para el año 1400 se estima en 375 millones de habitantes.

A partir de entonces, y pese a catástrofes demográficas tan graves como la que representó la conquista de América, cuya población se redujo, posiblemente, de unos 50 millones de habitantes a principios del siglo Xvı, a unos 10 a finales del mismo, la población mundial inició un proceso de crecimiento cada vez más acelerado, que alcanzó su máximo en las últimas décadas del siglo xx. Para el año 1500, la cifra anterior a la peste negra se había recuperado, con algo más de 450 millones de habitantes; tres siglos después, en 1800, la cifra se había duplicado, alcanzando los 900 millones. En sólo un siglo más se alcanzó poco menos de una nueva duplicación, con unos 1,600 millones de habi-

3 José Ángel García De Cortázar y J. A. Sesma Muñoz, Historia de la Edad Media. Una síntesis interpretaitva, Madrid, Alianza, 1997, pág. 226; y Vallín, op. cil. pág. 63.

4 Cfr. García y Sesma, op. cit., pág. 225.

5 Chaunu, op. cil., págs. 116-118. 


\section{Cundro 1}

Población mundial (en mlliones) en diversas épocas

\begin{tabular}{|c|c|c|c|}
\hline Época & \multicolumn{2}{|c|}{$\begin{array}{l}\text { Población } \\
\text { estimada }\end{array}$} & Observaciones \\
\hline$-10,000$ & & $5 ?$ & Neolítico, inicio de agricultura y ganadería. \\
\hline$-4,000$ & $15 ?$ & & Avances en allarería, metalurgia, ciudades. \\
\hline$-1,000$ & $30 ?$ & & \\
\hline-400 & 153 & & \\
\hline-200 & 225 & & \\
\hline 0 & 252 & $300 ?$ & $\begin{array}{l}\text { Fecha oficial del nacimiento de Cristo. Apogeo del } \\
\text { Imperio Romano con Augusto. }\end{array}$ \\
\hline 200 & 257 & & Decadencia del Imperio. \\
\hline 400 & 206 & & Invasiones báibaras; caída del Imperio. \\
\hline 600 & 205 & & Probable mínimo poblacional. \\
\hline 800 & 224 & & $\begin{array}{l}\text { Reorganización política de Europa; inicio de la moder- } \\
\text { nidad; recuperación demográfica. }\end{array}$ \\
\hline 1000 & 340 & & \\
\hline 1200 & 400 & & Auge europeo, escolástica, gólico, universidad. \\
\hline 1340 & 442 & & Máximo hislórico antes de la peste negıa. \\
\hline 1400 & 375 & & Reinicio de crecimiento. \\
\hline 1500 & 461 & & Despoblamiento de América por la conquista. \\
\hline 1600 & 578 & & Inicios de la ciencia moderna. \\
\hline 1700 & 771 & & Inicio de la revolución industrial. \\
\hline 1800 & 907 & $978 ?$ & Explosión demográfica emropea, más lenta. \\
\hline 1900 & 1,610 & $1,650 ?$ & $\begin{array}{l}\text { Avances científicos; descolonización; guerras; desa- } \\
\text { rrollo económico; explosión demográfica. }\end{array}$ \\
\hline 2000 & 6,080 & $6,055 ?$ & \\
\hline
\end{tabular}

Fuentes: Cohen, 1981, págs. 65; Vallín, 1995, pág. 63; Chaunu, 1982, págs. 51; Encyclopaedia Brilannica 1979; Celbard, Haub y Kent, 1999.

tantes. Y cien años más tarde, en nuestros días, la cifra anterior no se duplicó, sino que se multiplicó por cuatro, llegando a los 6,000 millones. El cuadro 1 sintetiza la evolución anterior. 
La aceleración del ritmo de crecimiento de la población durante los últimos siglos, especialmente el que acaba de terminar, es evidente. Tras decenas de miles de años de un aumento lento e irregular, la población del planeta llegó a unos 225 millones de personas, alrededor de 200 años antes de la era cristiana; luego, durante los mil años siguientes, hasta el 800 después de Cristo, la población volvió a aumentar y disminuir, para acabar ese milenio en un nivel prácticamente idéntico al inicial: 224 millones de habitantes.

El contraste que representa el siguiente milenio, del año 800 al 1800 después de Cristo, es claro: en lugar de mantenerse estacionaria, la población se multiplicó por cuatro en mil años, pasando de 224 a 907 millones. Se trata de un nuevo período en la historia de la humanidad, precisamente en la aurora del mundo moderno, marcada simbólicamente por la coronación de Carlo Magno como emperador del Sacro Imperio Romano Germánico el día de navidad del año 800, cuando el Imperio Romano de Occidente era ya un lejano recuerdo, y mientras el Imperio Bizantino, cada vez más helenizado y oriental, resistía con dificultad los embates de un joven y pujante Islam, que ya tenía casi un siglo de controlar desde los Pirineos hasta el Ganges, pasando por el norte de África. Durante los dos últimos siglos la tendencia se profundiza: en sólo cien años durante el siglo xIx, la población mundial casi se duplicó, pasando de 907 a 1,610 millones; y en otro siglo, el xx, la cifra se multiplicó de nuevo casi por cuatro, rebasándose la cifra de 6,000 millones en el año 2000.

\section{LA POBLACIÓN EN DIVERSAS REGIONES}

El comportamiento demográfico del conjunto de la población del planeta que se acaba de resumir, resulta, obviamente, de la combinación de los procesos demográficos que ocurrieron en las diversas regiones, los que no necesariamente fueron similares. Si bien a lo largo de la historia de la tierra se han presentado fenómenos de 
alcance planetario, que impactaron a todas las poblaciones vivientes, incluidas las humanas, seguramente con mayor frecuencia los factores que incidieron en el desarrollo de la población humana en cada región presentaron diferencias importantes. Esto resulta claro si se tiene en cuenta que esos factores incluyen elementos con una clara delimitación regional: climáticos, como sequías o heladas que se reflejan en épocas de bonanza o hambrunas; de salud, como la difusión de enfermedades epidémicas; y políticos, como guerras, conquistas y revoluciones.

Como se ha apuntado, la información demográfica sobre épocas antiguas existe principalmente para China y los principales países europeos, en tanto que para otros lugares sólo se dispone de cifras derivadas de estimaciones muy aproximadas y debatidas. Otra dificultad que se encuentra si se quieren comparar poblaciones a lo largo de períodos de tiempo prolongados es la que se deriva de la cambiante definición de las grandes entidades sociopolíticas y sus fronteras. Con las reservas del caso, el cuadro 2 presenta la evolución demográfica de algunas entidades geopolíticas importantes. Se enfatizan los conjuntos actuales, asociándolos, en forma aproximada, con conjuntos anteriores con los que se relacionan. Como elementos de comparación se incluyen dos importantes conjuntos de otras épocas: el Imperio Romano y el Imperio Austro-húngaro.

Puede apreciarse con facilidad el cambio de escala que se ha dado: al terminar el siglo xx, cada uno de los dos conjuntos más poblados (China e India, con los países vecinos que en algunos momentos de la historia pertenecieron a una entidad mayor que los aglutinaba) tienen una población cuatro veces mayor que la que tenía el mundo entero mil años antes, y muy superior a la del planeta apenas 200 años atrás. Cualquiera de los países europeos importantes (que, por mucho, ya no son los mayores del mundo) tienen hoy una población similar a la de los dos grandes imperios de los inicios de la era cristiana: el romano y el chino. Resulta impresionante, por otra parte, el crecimiento de nuevos gigantes, como Estados Unidos y Brasil, en comparación con las exiguas 


\begin{tabular}{|c|c|c|c|c|c|c|c|c|c|c|}
\hline \multicolumn{11}{|c|}{ Cuadro 2} \\
\hline \multirow[t]{2}{*}{ CONJUNTO } & \multicolumn{10}{|c|}{ POBLACIÓN ESTIMADA (EN MILLONES) } \\
\hline & 0 & 500 & 1000 & 1340 & 1500 & 1600 & 1800 & 1920 & 1950 & 1999 \\
\hline Imperio Romano & 54 & 30 & - & - & - & - & - & - & - & - \\
\hline Imperio Austro-húngaro & - & - & - & - & - & - & 22 & $44^{*}$ & - & - \\
\hline Imperio Chino (China Continental con & & & & & 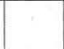 & & & & & \\
\hline Mongolia-Taiwán-Hong-Kong-Mlacao) & 57.7 & & 120 & 55 & & 60 & $221 ?$ & 480 & 571 & 1276 \\
\hline India (Con Pakistán y Bangladesh) & - & - & - & 一 & - & - & $150 ?$ & 304 & 434 & 1277 \\
\hline Nlemania (Oriental y Occidental) & - & - & - & 11 & - & - & 21 & 49.3 & 68.4 & 82.2 \\
\hline Reino Unido (C. Bretaña e lrlanda) & - & 0.5 & 1.0 & 4 & - & - & 11 & 43.7 & 50.6 & 58.7 \\
\hline Espuña y Portugal & - & - & - & 8.5 & 一 & - & 14.4 & 27.2 & 36.3 & 49.5 \\
\hline Francia & - & 3 & - & 19 & - & - & 27.3 & 38.8 & 41.7 & 58.9 \\
\hline Italia & $\longrightarrow$ & 一 & - & 9.3 & $一$ & - & 18.1 & 37.0 & 46.6 & 57.3 \\
\hline Imperio Ruso-URSS-Federación Rusa & - & - & - & 8 & - & - & 29 & 155 & 180 & 147 \\
\hline Estados Unidos & - & - & - & - & - & 1 & 6.3 & 76 & 151 & 276 \\
\hline Mesoamérica (México) & $一$ & - & - & - & $25 ?$ & $5-6$ & 5.8 & 14 & 25 & 96 \\
\hline Imperio Inca (Perú-Bolivia-Ecuador) & - & - & - & - & $15 ?$ & $3-4$ ? & 4 ? & 9.1 & 14.3 & 45.7 \\
\hline Brasil & - & - & - & - & $1-2$ ? & $1-2 ?$ & 3 & 27.6 & 52.3 & 168 \\
\hline TO'TAL, MUNDIAL & 252 & 207 & $3+10$ & 442 & 461 & 578 & 907 & 1860 & 2515 & 5978 \\
\hline
\end{tabular}

* 1914

Fuentes: Chaunu, 1982; Kinder y Ililgemann, 1978; ONU, 1967.

poblaciones de las respectivas regiones en el pasado. El caso de Rusia es distinto, con un crecimiento importante en el siglo XIX, seguido por una evolución desigual durante el xx.

\section{LA POBLACIÓN DE PÁ́SES Y CIUDADES EN EL SICLO XX Y EL XXI}

Como se ha comenzado a percibir en los apartados anteriores, desde un punto de vista demográfico el siglo xx, especialmente en su segunda mitad, constituye un período completamente excepcional en la historia de la humanidad, con un crecimiento 
mucho mayor al experimentado en cualquier época anterior. Debe señalarse que, a diferencia de otras épocas, las causas de esta explosión no son principalmente climáticas o coyunturales; en esta ocasión es obvia la rápida reducción de la mortalidad en general, y de la infantil en especial, debido, a su vez, a las profundas transformaciones de las condiciones de vida que han traído consigo los avances científicos, tecnológicos, económicos y educativos.

El cuadro 3 ofrece una imagen más detallada que muestra, a lo largo del siglo xx, la forma en que creció la población de diversos países, que se presentan en el orden que ocupaban en 1999. Es notable el contraste entre los grandes países europeos, cuya población creció relativamente poco (Alemania, Francia, Gran Bretaña e Italia, así como Rusia, si bien en este caso las cifras corresponden a la URSS hasta 1990, en tanto que en 1999 son las de la Federación Rusa, por lo que Ucrania aparece hasta este último año) y los países de Asia, África y América Latina, cuya población se incrementa en forma impresionante, multiplicándose varias veces.

\section{Cuadro 3}

Población de diversos Países duRANTE El SIGLO XX

\begin{tabular}{|c|l|r|r|r|r|r|r|r|r|r|}
\hline \multirow{2}{*}{ LUGAR } & \multicolumn{3}{|c|}{ País } & \multicolumn{6}{|c|}{ POBLACIÓN (MILLONES) ESTIMADA } \\
\cline { 3 - 10 } & & 1920 & 1930 & 1940 & 1950 & 1960 & 1970 & 1980 & 1990 & 1999 \\
\hline $1^{\circ}$ & China a & 475.0 & 500.0 & 530.0 & 558.2 & 654.5 & 771.8 & 994.4 & 1134 & 1267 \\
\hline $2^{\circ}$ & India b & 250.5 & 278.0 & 317.0 & 352.7 & 427.8 & 543.1 & 673.2 & 849.5 & 998.1 \\
\hline $3^{\circ}$ & Eslados Unidos & 106.8 & 123.6 & 132.6 & 152.3 & 180.7 & 204.9 & 227.3 & 250.9 & 276.2 \\
\hline $4^{\circ}$ & Indonesia & 52.3 & 60.8 & 70.5 & 75.5 & 92.7 & 119.5 & 146.2 & 181.6 & 209.3 \\
\hline $5^{\circ}$ & Brasil & 27.6 & 33.7 & 41.5 & 52.9 & 71.5 & 95.2 & 118.7 & 150.2 & 168.0 \\
\hline $6^{\circ}$ & Pakistán & 21.0 & 23.6 & 28.3 & 36.5 & 45.9 & 60.5 & 82.2 & 113.7 & 152.3 \\
\hline $7^{\circ}$ & Fed. Rusa c & 155.3 & 179.0 & 195.0 & 180.1 & 214.3 & 242.8 & 266.7 & 148 & 147.2 \\
\hline $8^{\circ}$ & Bangladesh & 33.3 & 35.6 & 42.0 & 41.0 & 51.4 & 67.7 & 90.1 & 113.2 & 126.9 \\
\hline $9^{\circ}$ & Japón & 56.0 & 64.5 & 72.0 & 83.6 & 94.1 & 104.3 & 116.6 & 123.5 & 126.5 \\
\hline
\end{tabular}




\begin{tabular}{|c|c|c|c|c|c|c|c|c|c|c|}
\hline $10^{\circ}$ & Nigeria & 22 & 25 & 29 & 34.3 & 42.9 & 55.1 & 84.7 & 117.5 & 121 \\
\hline $11^{\circ}$ & México & 14.4 & 16.6 & 19.8 & 26.6 & 36.4 & 50.3 & 67.5 & 86.2 & 97.4 \\
\hline $12^{\circ}$ & Alemania d & 49.3 & 55.7 & 59.8 & 68.4 & 72.7 & 77.8 & 77.8 & 77.3 & 82.2 \\
\hline $13^{\circ}$ & Vielnam e & 15.0 & 17.5 & 21.0 & 24.6 & 30.2 & 39.1 & 54.2 & 66.5 & 78.7 \\
\hline $14^{\circ}$ & Filipinas & 10.6 & 13.1 & 16.5 & 21.0 & 27.6 & 37.6 & 47.9 & 61.4 & 74.5 \\
\hline $15^{\circ}$ & Corea $f$ & 17.3 & 20.4 & 23.5 & 30.0 & 35.2 & 45.2 & 56.3 & 64.4 & 70.2 \\
\hline $16^{\circ}$ & Egipto & 13.3 & 14.8 & 16.9 & 20.5 & 25.9 & 33.3 & 39.8 & 52.1 & 67.2 \\
\hline $17^{\circ}$ & Irán & 11.0 & 12.4 & 14.0 & 16.9 & 21.6 & 28.4 & 38.1 & 56.9 & 66.8 \\
\hline $18^{\circ}$ & Turquía & 13.0 & 15.1 & 17.7 & 20.9 & 27.5 & 35.2 & 45.4 & 56.3 & 65.5 \\
\hline $19^{\circ}$ & Eliopráa & 11.0 & 12.5 & 14.5 & 16.7 & 20.0 & 24.9 & 31.5 & 51.2 & 61.1 \\
\hline $20^{\circ}$ & Tailandia & 9.5 & 11.8 & 15.3 & 20.0 & 26.4 & 35.7 & 46.5 & 5.8 .8 & 60.9 \\
\hline $21^{\circ}$ & Francia & 38.8 & 41.2 & 41.3 & 41.7 & 45.7 & 50.7 & 53.5 & 56.5 & 58.9 \\
\hline $22^{\circ}$ & Cran Bretaña & 43.7 & 45.9 & 48.2 & 50.6 & 52.6 & 55.5 & 55.9 & 57.5 & 58.7 \\
\hline $23^{\circ}$ & Italia & 37.0 & 40.3 & 43.9 & 46.8 & 50.2 & 53.6 & 56.9 & 57.6 & 57.3 \\
\hline 24 & Ucrania & $\longrightarrow$ & - & 一 & $\longrightarrow$ & 一 & - & $\longrightarrow$ & 52 & 50.7 \\
\hline $25^{\circ}$ & Rep. Dem. Congog & 10 & 10.5 & 11.5 & 12.5 & 14.1 & & 28.3 & 35.6 & 50.3 \\
\hline $26^{\circ}$ & Myanmar h & 13.1 & 14.3 & 16.1 & 18.4 & 22.3 & 27.7 & 33.3 & 41.6 & 45.1 \\
\hline $27^{\circ}$ & Colombia & 6.1 & 7.3 & 9.1 & 11.7 & 15.9 & 22.1 & 26.7 & 32.8 & 41.6 \\
\hline $28^{\circ}$ & Sudáfrica & 6.8 & 8.5 & 10.4 & 12.5 & 15.9 & 21.5 & 29.3 & 35.9 & 39.9 \\
\hline $29^{\circ}$ & España & 21.2 & 23.4 & 25.8 & 27.9 & 30.3 & 33.8 & 37.4 & 39.3 & 39.6 \\
\hline $30^{\circ}$ & Polonia & 26.0 & 29.5 & 31.5 & 24.8 & 29.6 & 32.5 & 35.8 & 38.0 & 38.7 \\
\hline
\end{tabular}

a: sin Hong Kong, Macao, Mongolia y Taiwán.

b): sin Pakistán ni Bangladesh.

c: hasta 1989 (1990) los datos son de la URSS.

d: Oriental y Occidental.

e: Norte y Sur.

f: Norte y Sur.

g: antes Zaire.

h: antes Birmania.

Fuentes: ONU, 1967; Anuarios El Pais.

El cuadro 4 presenta la misma realidad de otra manera, ordenando los 25 países más poblados del planeta a lo largo del siglo xx, a intervalos de 20 años. La primera observación que se impone es la relativa a la permanencia de China y la India en los dos 
primeros lugares; las proyecciones que se presentarán más adelante muestran que tal situación se mantendrá en el año 2025 pero que el orden se invertirá en el 2050, cuando la población de la India superará a la de China, si bien hay opiniones recientes en el sentido de que las estadísticas chinas subestimarían considerablemente la población del coloso oriental. ${ }^{6}$ El cuadro 2 ha mostrado también que, si se cuenta la población conjunta de la India, Pakistán y Bangladesh, que hasta la independencia de la región en 1948 formaban una unidad, el conjunto tenía ya, en 1999, más habitantes que China.

La otra observación obligada se refiere al retroceso de los grandes países europeos (incluyendo a Rusia, Alemania, Gran Bretaña, Francia, Italia, Polonia y España, e incluso Yugoslavia y Rumania) que a principios del siglo ocupaban lugares destacados en la jerarquía demográfica mundial, y a fines del mismo fueron reemplazados por un creciente número de países del tercer mundo. Las proyecciones que se presentan en el siguiente apartado muestran la continuación de esta tendencia.

\section{Cuadro 4}

LOS 25 PAíSES MÁS POBLAdOS DEL MUNDO EN EL SIGLO XX

\begin{tabular}{|c|c|c|c|l|l|}
\hline LUGAR & 1920 & 1940 & 1960 & 1980 & 1999 \\
\hline $1^{\circ}$ & China & China & China & China & China \\
\hline $2^{\circ}$ & India & India & India & India & India \\
\hline $3^{\circ}$ & URSS & URSS & URSS & URSS & USA \\
\hline $4^{\circ}$ & USA & USA & USA & USA & Indonesia \\
\hline $5^{\circ}$ & Japón & Japón & Japón & Indonesia & Brasil \\
\hline $6^{\circ}$ & Indonesia & Indonesia & Indonesia & Brasil & Pakistán \\
\hline $7^{\circ}$ & Alemania & Alemania & Alemania & Japón & Fed. Rusa \\
\hline
\end{tabular}

- Marc Mangin, "La population chinoise croii. vieillit et se masculinise", Le Monde. Dossiers \& Documents, $N^{\circ} 277$, juin, pág. 8. 


\begin{tabular}{|c|l|l|l|l|l|}
\hline $8^{\circ}$ & C. Bretaña & G. Brelaña & Brasil & Bangladesh & Bangladesh \\
\hline $9^{\circ}$ & Francia & Italia & G. Brelaña & Nigeria & Japón \\
\hline $10^{\circ}$ & Italia & Bangladesh & Bangladesh & Pakistán & Nigeria \\
\hline $11^{\circ}$ & Brasil & Brasil & Italia & Alemania & México \\
\hline $12^{\circ}$ & Polonia & Francia & Pakistán & México & Alemania \\
\hline $13^{\circ}$ & España & Polonia & Francia & Italia & Vietnam \\
\hline $14^{\circ}$ & Nigeria & Nigeria & Nigeria & Corea & Filipinas \\
\hline $15^{\circ}$ & Corea & Pakistán & México & G. Bretaña & Corea \\
\hline $16^{\circ}$ & Vietnam & España & Corea & Vietnam & Egipto \\
\hline $17^{\circ}$ & México & Corea & España & Francia & Irán \\
\hline $18^{\circ}$ & Egipto & Vietnam & Vietnam & Filipinas & Turquía \\
\hline $19^{\circ}$ & Birmania & México & Polonia & Tailandia & Etiopía \\
\hline $20^{\circ}$ & Turquía & Tuııuía & Filipinas & Turquía & Tailandia \\
\hline $21^{\circ}$ & Checoeslov. & Egipto & Turquía & Egipto & Francia \\
\hline $22^{\circ}$ & Yugoslavia & Filipinas & Tailandia & Irán & G. Bretaña \\
\hline $23^{\circ}$ & Rumania & Yugoslavia & Egipto & España & Italia \\
\hline $24^{\circ}$ & Etiopía & Birmania & Birmania & Polonia & Ucrania \\
\hline $25^{\circ}$ & Irán & Tailandia & Irán & Birmania & R. D. Congo \\
\hline
\end{tabular}

Fuente: Las mismas del cuadro 3.

El cuadro siguiente muestra la continuación de las tendencias mencionadas, con base en las proyecciones de la población por país al año 2025 y, parcialmente, al 2050. En el primer caso se presentan dos cifras, que corresponden a dos proyecciones diferentes, similares pero no idénticas, como puede apreciarse. Para el año 2050 solamente se mencionan los países.

Una perspectiva complementaria es la que se refiere al crecimiento de las principales ciudades del mundo. En este caso las diferencias de ritmo de crecimiento son todavía más marcadas, dado que, en la escala urbana, además del crecimiento natural, la migración del campo a la ciudad puede tener un peso considerable, a diferencia de lo que sucede con los países, que ven aumentar su población fundamentalmente por la diferencia entre 


\section{Cuadro 5}

Los Países Más Poblados Del Mundo en 2025 y 2050

\begin{tabular}{|c|c|c|c|c|}
\hline \multicolumn{3}{|c|}{2025} & \multicolumn{2}{|l|}{2050} \\
\hline PAÍS & $\mathrm{POB}$ & ÓN & PAÍS & POBLACIÓN \\
\hline China & $1,480.4$ & $1,561.4$ & India & 1,949 \\
\hline India & $1,330.4$ & $1,414.3$ & China & 1,389 \\
\hline USA & 325.6 & 335.1 & USA & \\
\hline Indonesia & 273.4 & 287.2 & Pakistán & \\
\hline Pakistán & 263 & 224.5 & Indonesia & \\
\hline Brasil & 217.9 & 217.9 & Nigeria & \\
\hline Nigeria & 183 & 203.4 & Brasil & \\
\hline Bangladesh & 178.8 & 177.0 & Bangladesh & \\
\hline Fed. Rusa & 137.9 & 138.1 & Eliopía & \\
\hline México & 130.2 & 140.8 & Rep. D. Congo & 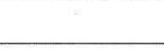 \\
\hline Japón & 121.2 & 120.9 & Filipinas & \\
\hline Etiopía & 115.4 & 98.8 & México & \\
\hline Filipinas & 108.3 & 111.5 & Vietnam & \\
\hline Vietnam & 108 & 109.9 & Egiplo & \\
\hline Rep. D. Congo & 104.8 & 105.7 & Irán & \\
\hline Egipto & 95.6 & 95.8 & Tailandia & \\
\hline Irán & 94.5 & 97.3 & Myanmar & \\
\hline Turquía & 87.9 & 88.0 & Fed. Rusa & \\
\hline Corea & 85.9 & 78.2 & Japón & \\
\hline Alemania & 80.2 & 79.9 & & \\
\hline Tailandia & 72.7 & 73.0 & & \\
\hline Francia & 61.7 & 64.2 & & \\
\hline Gran Bretaña & 60.0 & 62.6 & & \\
\hline Colombia & 59.8 & 58.3 & & \\
\hline Myanmar & 58.1 & 68.1 & & \\
\hline
\end{tabular}

Fuente: Vallín, 1995. Population Reference Bureau, 1999. 
natalidad y mortalidad, con las migraciones jugando normalmente un papel muy secundario, siendo los Estados Unidos la principal excepción histórica a esta regla.

El cuadro 6 muestra cómo las grandes capitales europeas están dejando rápidamente los primeros lugares entre las ciudades más pobladas a las metrópolis asiáticas, latinoamericanas y africanas, en las que grandes proporciones de los habitantes se hacinan en barriadas pobres.

\section{Cuadro 6}

LAS CIUDADES MÁS GRANDES DEL MUNDO, 1950-2015

\begin{tabular}{|l|c|l|c|l|c|}
\hline \multicolumn{2}{|c|}{1950} & \multicolumn{2}{c|}{ 1994 } & \multicolumn{2}{c|}{} \\
\hline Ciudad & Población & Ciudad & Población & Ciudad & Población \\
\hline Nueva York & 12.3 & Tokio & 26.5 & Tokio & 28.7 \\
\hline Londres & 8.7 & Nueva York & 16.3 & Bombay & 27.4 \\
\hline Tokio & 6.9 & Sao Paulo & 16.1 & Lagos & 24.4 \\
\hline París & 5.4 & México & 15.5 & Shanghai & 23.4 \\
\hline Moscú & 5.4 & Shanghai & 14.7 & Jakarta & 21.2 \\
\hline Shanghai & 5.3 & Bombay & 14.5 & Sao Paulo & 20.8 \\
\hline Essen & 5.3 & Los Ángeles & 12.2 & Karachi & 20.6 \\
\hline Buenos Aires & 5.0 & Beijing & 12.0 & Beijing & 19.4 \\
\hline Chicago & 4.9 & Calcuta & 11.5 & Dacca & 19.0 \\
\hline Calcuta & 4.4 & Seúl & 11.5 & México & 18.8 \\
\hline Osaka & 4.1 & Jakarta & 11.0 & Calcuta & 17.6 \\
\hline Los Ángeles & 4.0 & Buenos Aires & 10.9 & Delhi & 17.6 \\
\hline Beijing & 3.9 & Osaka & 10.6 & Nueva York & 17.6 \\
\hline Milán & 3.6 & Tianjin & 10.4 & Tianjin & 17.0 \\
\hline Berlín & 3.3 & Río de Janeiro & 9.8 & Manila & 14.7 \\
\hline
\end{tabular}

Fuentes: U. N. World Urbanization Prospects. The 1994 Revision. 1995. En Del Campo (1996) Cuadro 11, p. 52; Bernard (1999) p. 4. 


\section{LAS TENDENCIAS DEMOGRÁFICAS DE LARGO PLAZO}

La inercia de los fenómenos demográficos hace que, en horizontes de 20-30 años, las proyecciones sean bastante precisas y confiables, salvo cuando se presentan situaciones excepcionales; pero en períodos de tiempo mayores la incertidumbre aumenta, como puede verse en las siguientes proyecciones de la población al año 2050: la primera columna contiene la proyección media de la población según las Naciones Unidas, a principios de los años 90; las otras tres son las proyecciones más recientes, en las hipótesis alta, media y baja, del Population Reference Bureau de los Estados Unidos, en 1999.

\section{CuAdro 7}

Proyecciones de LA POBLACión mundial al AÑo 2050

\begin{tabular}{|l|r|r|r|r|}
\hline Bloque & 2050 & \multicolumn{3}{|c|}{2050} \\
\hline & & Alta & Media & Baja \\
\hline Asia & 5,599 & 6,316 & 5,268 & 4,312 \\
\hline Árrica & 2,265 & 2,102 & 1,766 & 1,467 \\
\hline América & 1,248 & 1,458 & 1,201 & 978 \\
\hline USA-Canadá & 326 & 464 & 392 & 324 \\
\hline América Latina & 922 & 994 & 809 & 654 \\
\hline Europa & 865 & 746 & 628 & 550 \\
\hline Oceanía & 41 & 52 & 46 & 36 \\
\hline Total & 10,018 & 10,674 & 8,909 & 7,343 \\
\hline
\end{tabular}

Fuentes: Vallín, 1995, Tabla IX, p. 96 (basada en ONU, World Population Prospects 1992; y Cellard, Haul, y Kent, 1999, Tablas 1 y 4, pp. 5 y 31.

En los casos de Asia y América, las proyecciones de 1992 se sitúan entre las variantes alta y media más recientes; en el caso de Oceanía entre las variantes media y baja; pero en los casos de Europa y África incluso las variantes altas de las proyecciones 
más recientes son inferiores a las proyecciones medias anteriores. En el caso de Europa, esto refleja la previsión de que la tendencia a la reducción de la natalidad del viejo continente se acentúe; en cuanto a África, en cambio, las proyecciones más recientes toman en cuenta el incremento en las tasas de mortalidad derivadas de la extensión de la pandemia que constituye el SIDA, cuyo impacto ha sido particularmente devastador en algunos países del África central y oriental al sur del Sahara.

Teniendo en cuenta, pues, la inevitable imprecisión de las mejores proyecciones, el cuadro siguiente ofrece una clara perspectiva del cambio de peso relativo de los continentes y algunos grandes conjuntos subcontinentales de mediados del siglo $\mathrm{xx}$ a mediados del xxIl, si las tendencias se mantienen. Puede apreciarse que Asia, América y Oceanía mantienen proporciones muy similares, mientras que Europa ve reducido su peso numérico en el 2150 a un tercio de lo que representaba en 1950 (7.3\% vs. $21.5 \%$ ) en tanto que la parte de Africa se multiplica por tres (pasando de $9.8 \%$ a $26.8 \%$ ). Es claro también el cambio en el peso relativo de las Américas, latina y del norte.

El cuadro 9 ofrece una perspectiva que se extiende dos siglos más atrás en el tiempo, hasta el año de 1750, cuando apuntaban ya con claridad las tendencias demográficas que dieron lugar al mundo actual, con el inicio de la transición demográfica en Europa. Sin considerar el caso marginal de Oceanía, los cuatro grandes continentes han visto cambios muy importantes en el peso relativo de sus poblaciones en el total mundial, y todo indica que los seguirán viendo en el siglo que apenas comienza.

En el siglo XVIII, habiéndose producido ya el colapso de la población americana como resultado de la conquista europea, el continente se recuperaba lentamente, mientras la población de la cuna de la humanidad, cinco o seis veces superior a la americana, se estancaba con el tráfico de esclavos, y en tanto Asia y Europa, desde muchos siglos antes los continentes más poblados, 
Cuadro 8

LA POBLACIÓN DE LOS GRANDES BLOQUES EN DOS SIGLOS

\begin{tabular}{|l|r|r|r|r|r|r|r|r|r|r|}
\hline Bloque & \multicolumn{2}{|c|}{1950} & 2000 & 2025 & 2050 & 2075 & 2100 & 2125 & 2150 \\
\hline & Absol. & $\%$ & Absol. & Absol. & Absol. & Absol. & Absol. & Absol. & Absol. & $\%$ \\
\hline Asia & $1,400.9$ & 55.0 & 3,692 & 4,900 & 5,599 & 5,825 & 5,978 & 6,075 & 6,145 & 53.2 \\
\hline China & 558.2 & & 1,310 & 1,540 & 1,521 & 1,475 & 1,405 & 1,400 & 1,389 & \\
\hline India & 352.7 & & 1,019 & 1,394 & 1,699 & 1,800 & 1,870 & 1,925 & 1,949 & \\
\hline Resto & 490 & & 1,363 & 1,966 & 2,379 & 2,550 & 2,703 & 2,750 & 2,807 & \\
\hline África & 250 & 09.8 & 857 & 1,582 & 2,265 & 2,650 & 2,931 & 3,025 & 3,090 & 26.8 \\
\hline América & 339 & 13.3 & 828 & 1,062 & 1,248 & 1,320 & 1,389 & 1,411 & 1,425 & 12.3 \\
\hline USA-Canadán & 172 & $(06.75)$ & 306 & 361 & 326 & 320 & 314 & 311 & 308 & $(02.66)$ \\
\hline Am. Latina & 167 & $(06.55)$ & 522 & 701 & 922 & 1,000 & 1,075 & 1,100 & 1,117 & $(09.67)$ \\
\hline Europa & 547 & 21.5 & 821 & 886 & 865 & 861 & 844 & 840 & 839 & 07.3 \\
\hline Ex-URSS & 180 & & 297 & 344 & 371 & 386 & 397 & 400 & 406 & \\
\hline Resto & 367 & & 524 & 542 & 494 & 475 & 447 & 440 & 433 & \\
\hline Oceanía & 11 & 00.4 & 31 & 41 & 41 & 41 & 41 & 41 & 41 & 00.4 \\
\hline Total & 2,548 & 100.0 & 6,229 & 8,471 & 10,018 & 10,697 & 11,183 & 11,392 & 11,540 & 100.0 \\
\hline
\end{tabular}

Fuentes: Vallín, 1995, Tabla IX, p. 96 (basada en ONU, World Population Prospects, 1992).

continuaban su crecimiento. En el siglo xIx, Europa dobla su población, mientras Asia únicamente conoce un incremento del orden de $50 \%$, y en tanto continúa el estancamiento de África y comienza el impresionante crecimiento de la población norteamericana, gracias sobre todo a la migración de los excedentes del crecimiento europeo que, en menor medida, alimentó también el crecimiento de la población de América Latina, particularmente en los países del Cono Sur.

En la primera mitad del siglo xx América del Norte mantiene el ritmo, pero se le añade América Latina y, en menor medida, Asia y África; en la segunda mitad son estos dos continentes, más el latinoamericano, los que ven incrementarse la proporción que les corresponde en el total, pero las tendencias para el siglo XXI 
apuntan en la dirección de la estabilización de la población americana, que sigue a la de Europa, que presenta un crecimiento reducido desde la primera mitad del siglo xx, en tanto que el avance asiático se hace más lento y el de África, incluso teniendo en cuenta la incidencia del sida, parece que seguirá siendo explosivo.

\section{Cuadro 9}

LA POBLACIÓN DE LOS GRANDES BLOQUES EN DOS SIGLOS

\begin{tabular}{|l|r|r|r|r|r|r|r|}
\hline Bloque & 1750 & 1800 & 1900 & 1950 & 2000 & 2025 & $2050^{*}$ \\
\hline Asia & 503 & 636 & 949 & 1,405 & 3,683 & 4,923 & 5,268 \\
\hline África & 106 & 107 & 133 & 221 & 784 & 1,290 & 1,766 \\
\hline América & 18 & 31 & 156 & 339 & 829 & 1,083 & 1,201 \\
\hline Del norte & 2 & 7 & 82 & 172 & 310 & 374 & 392 \\
\hline Latina & 16 & 24 & 74 & 167 & 519 & 709 & 809 \\
\hline Europa & 163 & 203 & 408 & 547 & 729 & 718 & 628 \\
\hline Oceanía & 1 & 1 & 4 & 11 & 30 & 41 & 46 \\
\hline Total & 791 & 978 & 1,650 & 2,521 & 6,055 & 8,054 & 8,909 \\
\hline
\end{tabular}

Fuente: Gellard, Haub y Kent, 1999, Talblas 1 y 4, pp. 5 y 31. Para el 2050, la proyección media.

\section{El CReCimiento ARITMÉTICO y EL GEOMÉTRICO}

Contra lo que pudiera pensarse, la explicación de cambios tan notables es sencilla, y su fundamento no es otra cosa que la diferencia entre el crecimiento aritmético y el geométrico, que ya había advertido desde el siglo xvill, y precisamente en relación con el crecimiento de la población, Thomas Malthus. ${ }^{7}$ El punto

7 Cfr. Anthony C. N Flew, "Introduction", En, Thomas R. Malthus, An Essay on the Principle of Population, Hardmonsworth, Penguin Books, 1970. $1^{\text {a }}$ ed. $1798 ; 2^{21} 1803$. 
que debe entenderse es la diferencia entre dos procesos de crecimiento, en uno de los cuales cada cierto tiempo (digamos cada año) se añade una cantidad constante, mientras que en el otro, la cantidad que se añade, en los mismos intervalos de tiempo, en lugar de ser siempre la misma, es una proporción constante de la cantidad anterior. En este segundo caso, aunque la proporción sea constante, como la base a la que se aplica va aumentando, también lo hace la cantidad que se añade periódicamente; se trata, pues, de un proceso en el que las cantidades absolutas que se aumentan son cada vez mayores, aunque la proporción que representan con respecto a la base sea idéritica, precisamente porque dicha base va aumentando. Por consiguiente el total que resulta después de cada incremento periódico va aumentando cada vez más.

El proceso se va acelerando con el paso del tiempo, a una velocidad que depende de la proporción de que se trate, en contraste con el primer caso, de incrementos constantes, en el que por mucho tiempo que pase los incrementos son iguales, y representan proporciones cada vez menores de la base a la que se añaden. Para aclarar las ideas, el cuadro siguiente presenta un ejemplo imaginario de dos países, ambos con una población inicial de 100. En uno de ellos tenemos una situación de incremento aritmético, que consiste en que cada año la población anterior aumenta en 1, indefinidamente. En el otro la población aumenta cada año en 1\%, o sea que el primer año el incremento es idéntico al del primer país, o sea 1, pero en los años sucesivos, al mantenerse constante la proporción del incremento, la cantidad absoluta del aumento va creciendo progresivamente.

Para evitar confusiones, recordamos que, prescindiendo de las migraciones, que en algunos casos pueden ser importantes, pero en conjunto se anulan unas a otras, el crecimiento demográfico se debe a la diferencia de la natalidad y la mortalidad. Si en un país nacen más personas de las que mueren en un período dado la población aumenta, y en caso contrario disminuye, cuando las defunciones son más numerosas que los nacimientos. Al 
manejar ciertas cifras de crecimiento (sea en términos absolutos o relativos, por ejemplo la de $1 \%$ en el ejemplo siguiente) y sumarlas a la cifra inicial, no se está suponiendo que la gente no se muere, sino simplemente que lo que se añade (una vez más, sea una cifra absoluta, sea una proporción) es precisamente la diferencia entre los nacimientos y las defunciones. Si se habla de crecimiento, se entiende que los primeros son más numerosos que las segundas y por ello la población aumenta en la forma expresada. Veamos pues lo que sucedería con la población de los dos ejemplos imaginarios al pasar el tiempo.

El período considerado en los ejemplos, de un siglo, no es especialmente largo en la escala histórica humana, y la tasa de crecimiento de $1 \%$ no es tampoco particularmente elevada en términos demográficos, si se tiene en cuenta que en el siglo Xx se observaron tasas de crecimiento natural de entre $3 \%$ y $4 \%$ en países latinoamericanos, africanos y asiáticos, durante varias décadas.

Con supuestos muy verosímiles, pues, el cuadro permite ver con claridad la diferencia entre un proceso de crecimiento aritmético, con aumentos anuales constantes en términos absolutos, y otro de tipo geométrico, con aumentos anuales proporcionalmente constantes, crecientes en términos absolutos. La diferencia parece insignificante en el corto plazo: a los diez años la población del primer ejemplo sería de 110, y la del segundo de 110.46. En un siglo la diferencia es ya muy importante: 200 contra 270.48 . Si el proceso continúa durante más tiempo las diferencias se hacen cada vez mayores: dos siglos más tarde, o sea trescientos años después de iniciado el proceso, el primer país ha llegado a una población de 4.00, en tanto que en el segundo se alcanzan ya casi 2,000 .

Los renglones sombreados en el cuadro llaman la atención sobre ciertos momentos importantes en el proceso: aquellos años en los que la población se duplica con respecto a la inicial. En el caso del país cuya población aumentara en forma aritmética, par- 
Cuadro 10

Dos eJemplos IMAGinaRios DE CRECIMIENTO

\begin{tabular}{|r|c|c|c|c|c|c|c|}
\hline \multicolumn{3}{|c}{ Crecimiento Aritmético } & \multicolumn{5}{|c|}{ Crecimiento Geométrico } \\
\hline Año & $\begin{array}{c}\text { Población } \\
\text { Inicial }\end{array}$ & $\begin{array}{c}\Delta \text { Anual } \\
\text { absoluto }\end{array}$ & $\begin{array}{l}\Delta \text { Anual } \\
\text { relativo }\end{array}$ & Año & $\begin{array}{c}\text { Población } \\
\text { Inicial }\end{array}$ & $\begin{array}{c}\Delta \text { Anual } \\
\text { absoluto }\end{array}$ & $\begin{array}{l}\Delta \text { Anual } \\
\text { relativo }\end{array}$ \\
\hline 0 & 100 & 1 anual & 0.01 & 0 & 100 & 1 & 0.01 \\
\hline 1 & 101 & 1 & 0.0099 & 1 & 101 & 1.01 & 0.01 \\
\hline 2 & 102 & 1 & 0.0098 & 2 & 102.01 & 1.02 & 0.01 \\
\hline 3 & 103 & 1 & 0.0097 & 3 & 103.03 & 1.03 & 0.01 \\
\hline 4 & 104 & 1 & 0.0096 & 4 & 104.06 & 1.05 & 0.01 \\
\hline 5 & 105 & 1 & 0.0095 & 5 & 105.1 & 1.05 & 0.01 \\
\hline 6 & 106 & 1 & 0.0094 & 6 & 106.15 & 1.06 & 0.01 \\
\hline 7 & 107 & 1 & 0.0093 & 7 & 107.21 & 1.07 & 0.01 \\
\hline 8 & 108 & 1 & 0.00926 & 8 & 108.286 & 1.08 & 0.01 \\
\hline 9 & 109 & 1 & 0.0092 & 9 & 109.37 & 1.09 & 0.01 \\
\hline 10 & 110 & 1 & 0.0091 & 10 & 110.46 & 1.1 & 0.01 \\
\hline 20 & 120 & 1 & 0.0083 & 20 & 122.02 & 1.22 & 0.01 \\
\hline 50 & 150 & 1 & 0.0067 & 50 & 164.46 & 1.65 & 0.01 \\
\hline 70 & 170 & 1 & 0.0059 & 70 & 200.67 & 2.01 & 0.01 \\
\hline 100 & 200 & 1 & 0.005 & 100 & 270.48 & 2.7 & 0.01 \\
\hline 140 & 240 & 1 & 0.0041 & 140 & 399 & 4 & 0.01 \\
\hline 170 & 270 & 1 & 0.0037 & 170 & 542.79 & 5.43 & 0.01 \\
\hline 200 & 300 & 1 & 0.0033 & 200 & 731.6 & 7.32 & 0.01 \\
\hline 210 & 310 & 1 & 0.0032 & 210 & 800 & 8 & 0.01 \\
\hline 280 & 380 & 1 & 0.00263 & 280 & 1,600 & 16 & 0.01 \\
\hline 300 & 400 & 1 & 0.0025 & 300 & 1,952 & 19.5 & 0.01 \\
\hline
\end{tabular}

tiendo de 100, y con incrementos anuales de 1, se necesitarían obviamente 100 años para que la población inicial se duplicara, llegando a 200; y para asistir a una nueva duplicación que lleve la población total a 400, deberá esperarse ahora 200 años; deberían pasar 400 años más para una duplicación más, para llegar a 800. 
En el caso de un país con crecimiento geométrico del $1 \%$ anual, en cambio, la población se duplicaría cada 70 años, como puede verse en los renglones sombreados de la parte derecha del cuadro anterior: a los 70 años la población total llega a los 200; a los 140 a los 400; a los 210 a los 800; y a los 280 a los 1,600; a los 350 años, cuando la población del país de crecimiento aritmética sería de 450, la del de crecimiento geométrico habría llegado a 3,200 y así sucesivamente.

Si en lugar de un crecimiento de $1 \%$ pensamos en tasas mayores, del $2 \%$, el $3 \%$ o, inclusive más, como se han dado de hecho históricamente en diversos lugares durante el siglo xx, el ritmo de crecimiento es, obviamente, más rápido y, obviamente, el tiempo que necesita una población para multiplicarse por dos es más reducido. Nuevamente en forma simulada, el cuadro 11 muestra varios ejemplos de ritmos de crecimiento diversos, indicando la población que tendrían al inicio del proceso varios países imaginarios con diferentes tasas de crecimiento, la que tendrían en 10 , 20 y 50 años, y el período de duplicación en cada caso.

\section{LAS CONSECUENCIAS DEL CRECIMIENTO DEMOGRÁFICO}

El carácter imaginario de los ejemplos utilizados en los cuadros 10 y 11, para ilustrar las nociones de crecimiento aritmético y geométrico, puede hacer pensar que la realidad dista mucho de tales simulaciones. Las cifras presentadas en apartados anteriores sobre la población del mundo, de diversas regiones y algunos países, muestran que no es así, sino que el crecimiento demográfico se comporta geométricamente, como debería esperarse, teniendo en cuenta que, al aumentar el número de personas que forman una población (y prescindiendo por el momento de los cambios en la proporción que representan en la pirámide poblacional los diversos grupos de edad) la proporción de naci- 


\section{Cuadro 11}

EJEMPLOS IMAGINARIOS DE CRECIMIENTO DEMOGRÁFICO

\begin{tabular}{|c|c|c|c|c|c|}
\hline \multirow{2}{*}{$\begin{array}{c}\text { Tasa } \Delta \\
\text { Anual }\end{array}$} & \multicolumn{4}{|c|}{ Poblactón en Miles de Habitantes } & \multirow{2}{*}{ Perído de } \\
\cline { 2 - 5 } & Inicial & A 10 AÑos & A 20 años & A 50 años & DUPLicación \\
\hline $0.0 \%$ & 10,000 & 10,000 & 10,000 & 10,000 & Indefinido \\
\hline $0.1 \%$ & 10,000 & 10,100 & 10,202 & 10,512 & 700 años \\
\hline $0.5 \%$ & 10,000 & 10,511 & 11,049 & 12,832 & 140 años \\
\hline $1.0 \%$ & 10,000 & 11,046 & 12,202 & 16,446 & 70 años \\
\hline $1.5 \%$ & 10,000 & 11,605 & 13,469 & 21,052 & 47 años \\
\hline $2.0 \%$ & 10,000 & 12,190 & 14,860 & 26,916 & 35 años \\
\hline $2.5 \%$ & 10,000 & 12,801 & 16,386 & 34,371 & 28 años \\
\hline $3.0 \%$ & 10,000 & 13,439 & 18,061 & 43,839 & 23.3 años \\
\hline $3.5 \%$ & 10,000 & 14,106 & 19,898 & 55,850 & 20 años \\
\hline $4.0 \%$ & 10,000 & 14,802 & 21,911 & 71,067 & 17.5 años \\
\hline
\end{tabular}

mientos y muertes no tiene por qué modificarse debido a ese crecimiento, sino que puede permanecer constante, si no se modifican otras condiciones (de alimentación, salud, conducta y otros). Y si esas proporciones se mantienen, el crecimiento se comportará geométricamente.

El cuadro 12, tomando como ejemplos los casos de México y del estado de Aguascalientes, en comparación con los de varios países, muestra como los ejemplos imaginarios se dan en la realidad.

Si comparamos la población de México en 1950 y en 1970, o en 1960 y 1980, podemos ver que en ambos casos se duplicó en 20 años, lo que quiere decir que durante las tres décadas en cuestión el país estuvo creciendo a una tasa aproximada de $3.5 \%$ anual, como resultado de una tasa de natalidad mayor a $4 \%$ y una de mortalidad inferior a $1 \%$, y eso sin contar el flujo migratorio de México hacia los Estados Unidos que, aunque no se dispone de datos exactos, en esos años tuvo ciertämente un saldo negativo para nuestro país de varios millones de personas. 
En contraste con lo anterior, el crecimiento de España y, más todavía, de Inglaterra y Bélgica, ha sido mucho menor. El resultado, en menos de un siglo, es que México, que en 1920 tenía sólo dos veces más habitantes que Bélgica, muchos menos que España y un tercio que Inglaterra, 80 años después es un gigante comparado con los tres países, incluido el otrora enorme Reino Unido.

Algo similar ocurre con el estado de Aguascalientes, que duplica ampliamente su población entre 1960 y 1980, entre 1970 y 1990, y de nuevo, ajustadamente, entre 1980 y el 2000, en tanto que Chipre, Luxemburgo, Malta, Martinica y Barbados crecen a ritmos mucho menores, con lo que el pequeño estado mexicano es ahora mucho mayor, en población, en comparación con cualquiera de dichos países.

Cuadro 12

CRecimiento REAL dE LA POBLACión DE VARIOS PAíSES

\begin{tabular}{|l|r|r|r|r|r|r|r|r|r|}
\hline País & 1920 & 1930 & 1940 & 1950 & 1960 & 1970 & 1980 & 1990 & 1999 \\
\hline México & 14,335 & 16,553 & 19,654 & 25,791 & 34,923 & 50,695 & 69,347 & 81,250 & 96,000 \\
\hline Inglaterra & 43,718 & 45,866 & 48,226 & 50,616 & 52,508 & 54,500 & 55,886 & 57,483 & 59,400 \\
\hline España & 21,390 & 23,668 & 25,878 & 27,977 & 30,528 & 34,041 & 37,681 & 39,500 & 39,600 \\
\hline Bélgica & 7,552 & 8,076 & 8,301 & 8,639 & 9,153 & 9,650 & 9,833 & 10,016 & 10,200 \\
\hline Aguascalientes & 108 & 133 & 162 & 188 & 243 & 338 & 519 & 720 & 950 \\
\hline Chipre & 319 & 357 & 413 & 497 & 573 & 600 & 620 & 701 & 760 \\
\hline Luxemburgo & 261 & 297 & 296 & 297 & 314 & 333 & 358 & 378 & 430 \\
\hline Malta & 210 & 240 & 270 & 312 & 329 & 350 & 343 & 353 & 390 \\
\hline Mautinica & 165 & 175 & 200 & 222 & 277 & 300 & 325 & 341 & 405 \\
\hline Barbados & 155 & 159 & 179 & 209 & 232 & 240 & 249 & 257 & 270 \\
\hline
\end{tabular}

Una consecuencia más de los diversos ritmos del crecimiento demográfico se refiere a la estructura de la pirámide de edades de la población. Cuando la diferencia entre las tasas de natalidad 
Cuadro 13

POBLACIÓN TOTAL Y POR GRUPOS DE EDAD EN VARIOS PAíSES DEL MUNDO (1987) Y ENTIDADES FEDERATIVAS (1990)

\begin{tabular}{|c|c|c|c|c|c|c|}
\hline \multirow[t]{2}{*}{ País o entidad } & \multirow[t]{2}{*}{ Población } & \multicolumn{5}{|c|}{ Porcentaje por Grupo de Edad } \\
\hline & & $0-14$ & $15-29$ & $30-44$ & $45-59$ & 606 más \\
\hline Estados Unidos & 243,773 & 22.6 & 27.4 & 19.1 & 15.2 & 15.7 \\
\hline Japón & 122,100 & 21.5 & 20.7 & 23.9 & 19.2 & 14.7 \\
\hline México & 81,323 & 43.0 & 27.8 & 14.9 & 8.4 & 5.8 \\
\hline Bélgica & 9,861 & 20.0 & 23.7 & 19.1 & 18.6 & 18.6 \\
\hline Estado de México & 9,816 & 45.0 & 27.7 & 15.9 & 7.2 & 4.2 \\
\hline Suecia & 8,387 & 17.9 & 20.7 & 22.2 & 16.1 & 23.1 \\
\hline Distrito Federal & 8,237 & 36.9 & 31.6 & 16.2 & 9.3 & 6.0 \\
\hline Suiza & 6,586 & 19.2 & 23.1 & 22.0 & 17.4 & 18.1 \\
\hline Veracruz & 6,215 & 42.3 & 27.1 & 15.8 & 9.0 & 5.8 \\
\hline Jalisco & 5,279 & 43.5 & 27.7 & 14.5 & 8.0 & 6.3 \\
\hline Dinamarca & 5,127 & 17.9 & 22.8 & 22.7 & 16.2 & 20.4 \\
\hline Aguascalientes & 720 & 43.5 & 28.0 & 13.7 & 8.8 & 6.0 \\
\hline Chipre & 719 & 25.0 & 26.6 & 20.1 & 13.8 & 14.5 \\
\hline
\end{tabular}

y las de mortalidad se mantiene en niveles reducidos durante períodos de tiempo prolongados (como sucedió durante milenios hasta que comenzó la primera transición demográfica, hacia el siglo XVIII en Europa) la pirámide se caracteriza por tener una base relativamente estrecha, que se amplía si la diferencia entre natalidad y mortalidad (especialmente infantil) aumenta, como sucedió en América Latina desde los años 50 del siglo xx, y en África un poco después.

Los países que se encuentran en esa situación de transición demográfica, cuando las tasas de mortalidad se han reducido y las de natalidad lo van haciendo más lentamente, además de crecer rápidamente, se caracterizan por tener una pirámide de una base más amplia, lo que significa que las personas de menor edad representan una proporción mayor que en los países que han dejado atrás la transición demográfica, cuya población es, en pro- 
medio, más vieja. El cuadro 13 nos presenta varios ejemplos de lo anterior, comparando la población total de México con las de Estados Unidos y Japón, y la de varios estados mexicanos con la de países de dimensiones totales similares, y luego haciendo la comparación de la proporción que representan los grupos de edad de 0 a 14 años (grupo al que característicamente pertenecen los alumnos de la educación básica); de 15 a 29 (grupo que incluye a los alumnos característicos de la educación media y superior); de 30 a 44; de 45 a 59; y de 60 años y más.

Resulta claro el peso mucho mayor que tiene, en la población de México y sus entidades, el grupo de 0 a 14 años, que representa en todos los casos cerca de la mitad del total (42.5\% a 45\%) mientras que en los países con los que se compara representa solamente del $17.9 \%$ al 22.6\%. En el grupo de 15 a 29 años las cifras mexicanas son también superiores a las de los demás países, pero con diferencias mucho menores, y en los grupos de mayor edad la situación se invierte.

Las implicaciones de lo anterior para los sistemas educativos son evidentes, y pueden verse en el cuadro 14, en el que se compara la matrícula en la enseñanza primaria, la media (incluyendo los niveles de secundaria y la llamada media superior) y la superior en México y varios de sus estados, en relación con los mismos países del cuadro anterior.

Puede apreciarse que, pese a tener una población total muy inferior a la de Japón al terminar la década de los años 80 del siglo xx, en México había muchos más niños en primaria que en el país oriental, y casi la mitad que en los Estados Unidos, a pesar de que la población total de México sólo representaba un tercio de la de aquel país.

De manera similar, pese a poblaciones totales muy semejantes, las entidades mexicanas tienen un número mucho mayor de niños en primaria en comparación con los países de referencia.

Las implicaciones del crecimiento demográfico en el campo de las políticas educativas son claras, y los cuadros anteriores las 
ponen de manifiesto: el esfuerzo que tiene que hacer un país para que sus habitantes puedan disfrutar de niveles adecuados de bienestar es muy diferente en condiciones de población relativamente estable, en comparación con la situación que se presenta en los casos de crecimiento acelerado. Y en el campo educativo el impacto del crecimiento es particularmente fuerte desde las primeras etapas de la transición demográfica, puesto que son precisamente los grupos de edad que requieren de educación básica los primeros en crecer.

El último cuadro de este trabajo nos permite precisar esta última idea. En él puede apreciarse cómo hasta 1980 la matrícula de primaria aumentó en México a un ritmo mayor inclusive al de la población total del país, dado que, además del incremento poblacional, al mismo tiempo el sistema educativo fue atendiendo a una proporción creciente de la demanda.

A partir de los años 80, México entró en una etapa más avanzada de su transición demográfica, al comenzar a notarse la reducción de la natalidad iniciada en la década anterior, tanto en forma espontánea como debido a los programas gubernamentales de control natal. A diferencia de lo que sucedía desde fines de los 40, cuando cada año nacían varios centenares de miles de niños más que el anterior, a partir de 1975 los nacimientos anuales se estabilizaron y comenzaron a disminuir poco a poco. Las sucesivas cohortes de niños que llegaron a la escuela a partir de 1982 reflejaron ese fenómeno en forma evidente: aunque la población todavía creció de manera significativa, puesto que la mortalidad seguía siendo muy baja (.4 ó .5\%) al predominar una población no vieja, y dado que la cobertura de $100 \%$ en el acceso a la primaria se alcanzó precisamente hacia 1982, el número total de alumnos de primaria en el país se ha mantenido prácticamente constante desde entonces hasta la fecha.

El cuadro 15 nos muestra cuál ha sido realmente la población total de México y la matrícula de niños en las escuelas primarias del país del año 1920 al 2000, y cuál habría sido la población en 


\section{Cuadro 14}

Matrícula ESCOLAR POR NIVEL EN VARIOS PAíses DEL MUNDO (1987) Y ENTIDADES FEDERATIVAS (1989)

\begin{tabular}{|l|r|r|r|r|}
\hline \multirow{2}{*}{ País o entidad } & \multirow{2}{*}{ POBLACIÓN } & \multicolumn{3}{|c|}{ MATRÍCULA POR NIVEL } \\
\cline { 3 - 5 } & & PRIMARIA & MEDIA & SUPERIOR \\
\hline Estados Unidos & 243,773 & 31,555 & 13,703 & 12,164 \\
\hline Japón & 122,100 & 10,226 & 11,456 & 2,597 \\
\hline México & 81,323 & 14,494 & 6,795 & 1,121 \\
\hline Bélgica & 9,861 & 768 & 1,077 & 102 \\
\hline Estado de México & 9,816 & 1,717 & 688 & 95 \\
\hline Suecia & 8,387 & 960 & 267 & 221 \\
\hline Distrito Federal & 8,237 & 1,134 & 918 & 254 \\
\hline Suiza & 6,586 & 406 & 619 & 117 \\
\hline Veracruz & 6,215 & 1,127 & 409 & 69 \\
\hline Jalisco & 5,279 & 969 & 410 & 100 \\
\hline Dinamarca & 5,127 & 415 & 484 & 124 \\
\hline Aguascalientes & 720 & 122 & 64 & 7.8 \\
\hline Chipre & 719 & 51 & 46 & 3.1 \\
\hline
\end{tabular}

los últimos años del siglo si se hubiera mantenido constante las tasas de crecimiento de 3.5\% anual desde 1950 no hasta 1980, sino hasta el 2000, y cual sería la matrícula de primaria si las tasas observadas de alrededor de $5.65 \%$ anual de 1950 a 1980 (lo que implica que el total se triplique cada 20 años) se hubieran mantenido sin cambios hasta el 2000.

Conclusión

Para concluir, se destacan dos ideas: la primera se refiere al fenómeno mismo del crecimiento demográfico de México durante la segunda mitad del siglo Xx. 
Cuadro 15

Población dE MÉXICO Y MATRíCULA DE PRIMARIA A LARGO DEL SIGLO XX

\begin{tabular}{|c|c|c|c|c|}
\hline \multirow{2}{*}{ FECHA } & \multicolumn{2}{|c|}{ POBLACIÓN TOTAL } & \multicolumn{2}{c|}{ MATRÍCULA PRIMARIA } \\
& $\Delta \mathbf{3 . 5} \% \mathbf{1 9 5 0}$ & \multicolumn{1}{c}{ Real } & $\Delta 5.65 \% 1980$ & Real \\
\hline 1920 & $14,335,000$ & $14,335,000$ & 868,000 & 868,000 \\
\hline 1930 & $16,553,000$ & $16,553,000$ & $1,300,000$ & $1,300,000$ \\
\hline 1940 & $19,654,000$ & $19,654,000$ & $1,961,000$ & $1,961,000$ \\
\hline 1950 & $25,791,000$ & $25,791,000$ & $3,030,000$ & $3,030,000$ \\
\hline 1960 & $36,380,753$ & $34,923,000$ & $5,402,000$ & $5,402,000$ \\
\hline 1970 & $51,318,645$ & $50.695,000$ & $9,248,000$ & $9,248,000$ \\
\hline 1980 & $72,390,016$ & $69,347,000$ & $14,628,000$ & $14,628,000$ \\
\hline 1990 & $102,113,270$ & $81,250,000$ & $27,750,000$ & $14,402,000$ \\
\hline 1995 & $121,278,530$ & $91,120,000$ & & $14,574,000$ \\
\hline 2000 & $144,040,850$ & $96,200,000$ & $43,884,000$ & $14,500,000$ \\
\hline
\end{tabular}

Por la dificultad de entender este tipo de procesos, y por el hecho de no haber conocido una realidad diferente, la inmensa mayoría de los casi 100 millones de mexicanos que actualmente habitamos el país no cae en la cuenta de lo excepcional que fue esa segunda mitad del siglo XX, en términos demográficos, no sólo a nivel nacional sino mundial.

Pocas personas entienden que el crecimiento natural (sin contar los flujos migratorios) que México experimentó de 1950 a 1980 no había tenido precedente alguno en toda la historia de la humanidad.

Si se entiende lo anterior, al analizar la situación educativa del país, por ejemplo, no deberá sorprender que se enfrenten problemas de calidad en la educación primaria. Haber logrado incrementar la cobertura de ese nivel (que pasó de poco más del $60 \%$ en 1959 a prácticamente 100\% en 1982), precisamente cuando la población del país, y más la de sus niños, crecía de manera explosiva, es un logro considerable, del que se puede estar satis- 
fecho, aún si la calidad se vio afectada. Y el que de principios de los 80 en adelante la matrícula de primaria se haya estabilizado constituye una oportunidad esperanzadora en lo que toca al mejoramiento cualitativo.

La segunda idea tiene que ver, de manera general, con el planteamiento hecho en la introducción, en lo relativo a la dificultad de muchas personas de entender fenómenos de crecimiento geométrico.

En 1981 terminó su trabajo un grupo formado por el parlamento de la Gran Bretaña para estudiar la problemática de la enseñanza de las matemáticas, con el énfasis puesto en la identificación de los aspectos de dicha ciencia que resultan más necesarios en la vida diaria de cualquier persona, y no sólo para los especialistas de ciertos campos. Uno de los estudios realizados en el marco de este trabajo encontró lo siguiente:

Otra característica revelada por el estudio fue una extendida incapacidad para entender los porcentajes. Muchos de los entrevistados dijeron que no los entendían o que nunca los usaban... Otros que dijeron ser capaces de calcular el $10 \%$ y, quizás, pero con dificultad, el 15\%, indicaron que no serían capaces de afrontar las dificultades que representaba calcular el $8 \%$ o el $12 \%$... Es claro que los políticos, administradores, hombres de negocios, periodistas y anunciantes, todos suponen que a la larga el público comprenderá las muchas afirmaciones que expresan comparaciones en términos de porcentajes. El estudio indica que esto está muy lejos de la realidad... Otra pregunta demostró que una estadística que normalmente se expresa en términos de porcentaje, la de la tasa de inflación, se entiende aún $\operatorname{menos...8}$

Si a muchas personas les resulta difícil entender un simple porcentaje, no es raro que menos todavía entiendan los procesos de crecimiento que implican la aplicación de tasas porcentuales en forma acumulativa a partir de cierta base, en períodos de tiempo

8 William H. Cockcroft, Sobre la enseñanza de las matemáticas, Madrid, Morata, 1985, págs. 11-12. 
prolongado, como es el caso del crecimiento demográfico o, de manera muy similar, el del producto per capita, que resulta de la diferencia entre el crecimiento del producto interno bruto y el de la población que lo genera y entre la que se distribuye (en forma que dista mucho de ser equitativa).

El análisis de cifras históricas reales y el de los ejemplos imaginarios que se han presentado podrá ayudar a mejorar la comprensión de este tipo de fenómenos. 